\title{
Hot carrier aging and its variation under use-bias: kinetics, prediction, impact on Vdd and SRAM
}

\author{
M. Duan ${ }^{1}$, J. F. Zhang ${ }^{1}$, A. Manut ${ }^{1}$, Z. Ji ${ }^{1}$, W. Zhang ${ }^{1}$, A. Asenov ${ }^{2}$, L. Gerrer ${ }^{2}$, D. Reid ${ }^{3}$, H. Razaidi ${ }^{2}$,

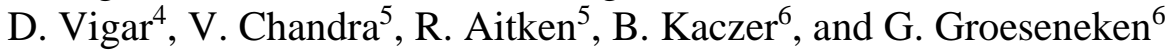 \\ ${ }^{1}$ School of Engineering, Liverpool John Moores University, Byrom Street, Liverpool L3 3AF, UK ( j.f.zhang@ljmu.ac.uk ) \\ ${ }^{2}$ Dept. Electronics and Electrical Engineering, University of Glasgow, UK. ${ }^{3} \mathrm{GSS}$, Glasgow, UK. \\ ${ }^{4} \mathrm{CSR}$, Cambridge CB4 OWZ, UK. $\quad{ }^{5}$ Arm R\&D, San Jose, USA. \\ ${ }^{6}$ IMEC, Leuven B3001, Belgium.
}

\begin{abstract}
As CMOS scales down, hot carrier aging (HCA) scales up and can be a limiting aging process again. This has motivated re-visiting HCA, but recent works have focused on accelerated HCA by raising stress biases and there is little information on HCA under use-biases. Early works proposed that HCA mechanism under high and low biases are different, questioning if the high-bias data can be used for predicting HCA under use-bias. A key advance of this work is proposing a new methodology for evaluating the HCA-induced variation under use-bias. For the first time, the capability of predicting HCA under use-bias is experimentally verified. The importance of separating RTN from HCA is demonstrated. We point out the HCA measured by the commercial SourceMeasure-Unit (SMU) gives erroneous power exponent. The proposed methodology minimizes the number of tests and the model requires only 3 fitting parameters, making it readily implementable.
\end{abstract}

\section{Introduction}

Recent results (Fig.1) show Hot Carrier Aging (HCA) can be severe for current/future CMOS nodes [1-7], because: (i) Channel length downscaling enhances HCA (Fig.2a). For some sub-30nm processes, HCA can be higher than BTI (Figs.1b\&2b); (ii) HCA can have larger time exponents (Figs.1b\&2b) [3-7] and its importance increases with aging. (iii) NBTI recovery [8-10] is higher than HCA (Fig.2c); (iv) Conventionally, the worst HCA occurs during switch near $\mathrm{Vg} \sim \mathrm{Vd} / 2$ and duty factor (DF) is typically low $(1 \sim 2 \%)[7,11]$. For modern CMOS, however, more damage occurs under $\mathrm{Vg}=\mathrm{Vd}$ (Fig. 3) $[3,6,7]$ and DF can be high. For example, during 'read 0' in a SRAM cell, one access nMOSFET can suffer HCA for $\sim 50 \%$ of time (Fig. 4).

The renewed HCA-threat has motivated its re-visit [1$7,12]$. It is reported aging mechanisms and time exponent, ' $n$ ' (Eq.1 in Table 1), are different under different stress biases $[1,6,7]$. 'n' can also vary with time (e.g. Fig.5) [2,4,5,7], challenging the lifetime, $\tau$, prediction based on Eq.1 that requires a constant ' $n$ ' $[11,13,14]$. The recent works have focused on bias-accelerated HCA [1-7,12] and there is little data on HCA under use-bias. For test engineers, two pressing questions are: can $\tau$ under use-bias still be predicted by the established JEDEC method based on Eq.1 and how to evaluate ' $\mathrm{n}$ ' correctly for HCA? A key advance of this work is answering them and finding the pitfalls for extracting ' $n$ '. For the first time, the capability of predicting HCA under use-bias is experimentally verified (Fig.6).

\section{Devices and Experiments}

nMOSFETs of MG/HK were made by an industrial process with $\mathrm{L} \times \mathrm{W}$ of $27 \times(90 \sim 900) \mathrm{nm}$ and use-Vdd of $0.9 \mathrm{~V}$. $\mathrm{Vd}=\mathrm{Vg}$ is chosen to represent stress, as Isub/Id has a deviceto-device variation (DDV) at stress-0 for nm-devices (Fig.7a) and it does not correlate with HCA (Fig.7b). All tests were at $125^{\circ} \mathrm{C}$

\section{A. Selecting parameter for extracting power exponent, ' $n$ ' \\ HCA was widely monitored by forward saturation current} shift under $\quad \mathrm{Vg}=\mathrm{Vd}=\mathrm{Vdd}, \quad \Delta \mathrm{Id} / \mathrm{Id} \_\mathrm{F}$, although reverse saturation current shift, $\Delta \mathrm{Id} / \mathrm{Id} \mathrm{R}$, and $\Delta \mathrm{Vth}(\mathrm{Vd} \leq 0.1 \mathrm{~V})$ also were used $[1-7,11,12]$. The problem is ' $n$ ' for $\Delta \mathrm{Id} / \mathrm{Id} F$ is larger than ' $n$ ' for $\Delta \mathrm{Id} / \mathrm{Id} \mathrm{R}$, leading to their incorrect crossover and errors in prediction at 10 years (Fig.8), highlighting the importance of ' $n$ '-accuracy. Under $\mathrm{Vg}=\mathrm{Vd}, \Delta \mathrm{Id} / \mathrm{Id} \_\mathrm{F}$ does not sense the HCA-defects above space charge region (Fig.8), resulting in an apparent larger ' $\mathrm{n}$ ', as simulated by subtracting a constant from real power law (inset of Fig.8). The ' $n$ ' extracted from the forward $\Delta \mathrm{Id} / \mathrm{Id}$ is erroneous. To capture all defects, $\Delta \mathrm{Vth}(\mathrm{Vd}=0.1 \mathrm{~V})$ should be used for extracting ' $\mathrm{n}$ ', as $\Delta V$ th_F $=\Delta V$ th_R (Fig.9). Once $\Delta V$ th is predicted, we propose evaluating $\Delta \mathrm{Id} / \mathrm{Id}_{-} \mathrm{F}$ and $\Delta \mathrm{Id} / \mathrm{Id}_{-} \mathrm{R}$ by using their measured relation with $\Delta \mathrm{Vth}$ (Fig.10).

\section{B. HCA acceleration}

SRAM often is used for qualifying new processes [15], where the access nMOSFETs suffer the worst HCA under $\mathrm{Vg} \approx \mathrm{Vd}$ (Figs.3\&4). HCA under use- $\mathrm{Vg}=\mathrm{Vd}$ must be predicted and we focus on it here. Under use-bias, Fig.11 shows that HCA is too low to establish its kinetics reliably within a practical time and acceleration is needed. One may accelerate HCA by raising $\mathrm{Vd}$ only [12] or both $\mathrm{Vg}$ and $\mathrm{Vd}$ with $\mathrm{Vg}=\mathrm{Vd}[1,6]$. Fig.12a confirms ' $\mathrm{n}$ ' is larger under $\mathrm{Vg}<\mathrm{Vd}$ than under $\mathrm{Vg}=\mathrm{Vd}[1,7]$, so that $\mathrm{Vg}<\mathrm{Vd}$ must not be used to predict HCA under use- $\mathrm{Vg}=\mathrm{Vd}$. When accelerated by $\mathrm{Vg}=\mathrm{Vd}$, ' $\mathrm{n}$ ' is bias-independent (Fig.12b) and should be used.

\section{DC versus $A C$}

Unlike NBTI(AC)<NBTI(DC) [8-10], the AC and DC HCAs agree well, regardless of frequency and duty-factor (DF) for the same equivalent stress time, i.e. DFxtime (Fig.13), confirming the frequency-independence [3]. DC will be used.

\section{Voltage-Step-Stress (VSS)}

The VSS technique recently developed for NBTI [16] allows extracting both ' $n$ ' and 'm' (Eq.1) from just one large 
device, reducing test numbers by $\sim 80 \%$, and will be adopted for HCA here. For an $\mathrm{L} \times \mathrm{W}=27 \times 900 \mathrm{~nm}$, stress under each $\mathrm{Vg}=\mathrm{Vd}$ lasted for $\mathrm{T}=1 \mathrm{ks}$ and biases were then stepped up (Fig.14), lifting HCA up from the power law (Fig.14c). Based on Eqs.1-3, HCA under a high $\mathrm{Vg}=\mathrm{Vd}$ was converted into a longer equivalent stress time under a low $\mathrm{Vg}=\mathrm{Vd}$ (Fig.14c) and $\Delta V$ th follows a power law well even when $\Delta V$ th $>150 \mathrm{mV}$, corresponding to $\Delta \mathrm{Id} / \mathrm{Id}>30 \%$ (Fig.10), well beyond the typical $10 \%$ HCA lifetime criterion and allowing reliable extraction of ' $n$ ' and 'm' (Fig.14c).

\section{Prediction}

A model is useful only if it can predict aging under usebias. The HCA predicted by the model extracted from the VSS data in Fig.14 agrees well with the test data in Figs.6b-g. The highest $\Delta$ Vth in Figs.6a\&14 is $\sim 2$-orders above $\Delta$ Vth under $0.9 \mathrm{~V}$ (Fig.6b), verifying its prediction capability. We emphasize the model was extracted from the data in Fig.6a only and the test data in Figs.6b-g were not used for fitting. The extracted model (Eq.1) can be used for evaluating HCA under any bias and time and for predicting lifetime and operation Vdd (Fig. 15).

\section{HCA in nm-width devices}

Unlike $\mathrm{L} \times \mathrm{W}=27 \times 900 \mathrm{~nm}, 27 \times 90 \mathrm{~nm}$ devices suffer from RTN-like within-a-device fluctuation (WDF) and large device-to-device variation (DDV) (Fig.16). To extract HCA kinetics, one has to use the smooth mean of 50 devices, but ' $\mathrm{n}$ ' depends on how data is taken (Fig.17). After a stress, $\Delta V$ th fluctuates and one can use its up-envelope (UE), lowerenvelope (LE) [17], or average over a period of time, e.g. $\sim 10 \mathrm{~ms}$ (Fig.17b), as a typical quasi-DC Source-Measure-Unit (SMU) does. The 'n' from UE and DC (inset of Fig. 17a) is smaller than the ' $n=0.29^{\prime}$ from a device of $W=900 \mathrm{~nm}$ (Fig.14c), but the ' $\mathrm{n}$ ' from LE agrees well with it. The smaller ' $\mathrm{n}$ ' for UE incorrectly takes it below LE when extrapolating (see the 'cross-over' in Fig. 17a).

To explain the difference in 'n', Figs.18a\&b show that $\mathrm{WDF}=(\mathrm{UE}-\mathrm{LE})$ does not increase with aging. It must originate from as-grown defects and should be excluded from aging kinetics, so that LE must be used for extracting ' $n$ '. LE_F and LE_R correlates (Fig.19a), but WDF_F and WDF_R does not (Fig.19b), supporting their different origins.

Since HCA-recovery is insignificant (Fig.2c), one may think it can be measured by a quasi-DC SMU [7,18]. This, however, gives an erroneous lower 'n' (Fig.17a) by including some as-grown WDF. Adding a constant to a power law leads to an apparent lower ' $n$ ' at short time and a variation of ' $n$ ' with time (inset, Fig.17a) [13].

\section{Statistic HCA}

The DDV of LE at different time (Figs.20a\&b) and voltage (Figs.20c\&d) follows the defect-centric distribution (Eqs.4\&5) well [19]. LE_mean of $50 \mathrm{~W}=90 \mathrm{~nm}$ devices agrees well with $\Delta \mathrm{V}$ th of one $\mathrm{W}=900 \mathrm{~nm}$ device (Fig.21a) and can be predicted by the same method (Figs.6\&14). After knowing LE mean, the standard deviation, $\sigma$, can be evaluated from its power law relation with the mean (Fig.21b).

\section{A. Impact on use-Vdd}

To have a yield corresponding to $\mathrm{i} \times \sigma, \Delta \mathrm{Id} / \mathrm{Id}=10 \%$ is required at $i \times \sigma$, resulting in smaller mean value (Fig.22a) and in turn lower use-Vdd (Fig.22b) for higher i. For a yield of $3 \times \sigma(99.7 \%)$, HCA-only and $\mathrm{HCA}+\mathrm{WDF}(\mathrm{Eqs} .6,7)$ reduces Vdd from its zero-spread value by $75 \mathrm{mV}$ and $100 \mathrm{mV}$, respectively.

\section{B. Impact on 6T-SRAM}

Assuming only one access nMOSFET suffered HCA and using the predicted HCA distribution at 10 years under $0.9 \mathrm{~V}$ for simulation [20], static write/read noise margin reduces/rises, respectively (Fig.23), as a weakened access nMOSFET is not in favor of write. Both the dynamic read (Figs.24a-c) and write (Figs.24d-f) access time deteriorates, since longer time is required through a weakened access nMOSFET. This demonstrates that the extracted HCA model can be incorporated into a compact simulator to evaluate the required margin for a specified yield.

\section{Conclusions}

As CMOS scales down, HCA scales up. For the first time, this work experimentally verifies that the HCA under use-Vdd can be predicted by the power law extracted from VSSmethod, provided that correct acceleration and ' $n$ '-evaluation are made. We point out the forward saturation $\Delta \mathrm{Id} / \mathrm{Id}$ and HCA measured by SMU gives erroneous ' $n$ ' for nm-width devices. The model requires only 3 fitting parameters (Eq.1), making it readily implementable.

\section{Acknowledgement}

The authors thank Plamen Asenov of GSS for his critical comments. This work was supported by the EPSRC of U.K. under the Grant No. EP/L010607/1.

\section{References}

[1] A. Bravaix et al, IRPS 2013, 2D.6.1-2D6.9.

[2] J. H. Stathis et al, IEDM 2014, p. 522.

[3] G. T. Sasse, IEEE Trans.Elec.Dev., 55, pp.3167-3174, 2008.

[4] A. J. Scholten et al, IEEE Trans.Elec.Dev., 58, pp.1-8, 2011.

[5] F. Cacho et al. IRPS 2014, 5D.4.1.

[6] A. Bravaix et al, IEDM 2011, p.622.

[7] M. Cho et al, IEEE Trans.Elec.Dev., 60, pp.4002-4007, 2013

[8] S. Ramey et al, IRPS 2014, XT.2.1.

[9] M. H. Chang and J. F. Zhang, J.Appl.Phys., 101, art. no. 024516, 2007.

[10] S. F. W. M. Hatta et al, IEEE Trans.Elec.Dev., 60, pp.1745-1753, 2013.

[11] JEDEC 2011 p.15.

[12] C. Liu et al, IEDM 2014, p 836.

[13] Z. Ji et al, IEDM 2013, p.413

[14] Z. Ji et al, IEEE Trans.Elec.Dev., 57, pp.228-237, 2010.

[15] U. Bhattacharya, Intel Tech. J. 2008, p.111.

[16] Z. Ji et al, IRPS 2014, GD-2.

[17] M. Duan et al, IEDM 2013, p.774.

[18] N. H. H. Hsu et al, IRPS 2012.

[19] L. M. Procel et al, IEEE Elec.Dev.Lett., 35, p. 1167-1169, 2014

[20] http://ptm.asu.edu/. 

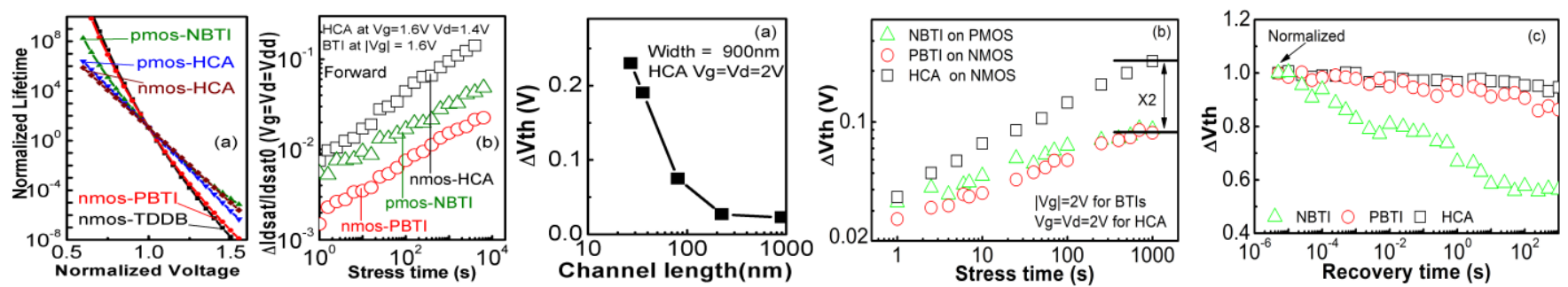

Fig 1 A comparison of Hot Carrier Aging (HCA) with Fig.2 (a) Downscaling L increases HCA. The stress was at $125^{\circ} \mathrm{C}$ for 1000 sec. (b) A comparison of of data from refs. [2] and [1], respectively.

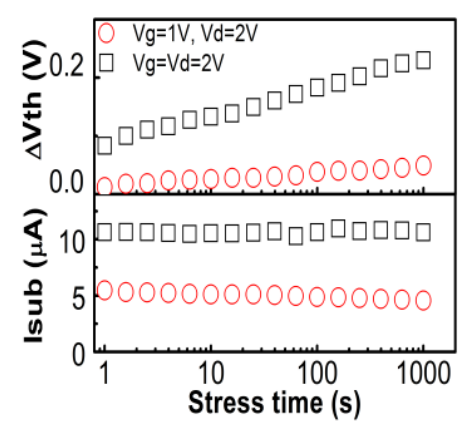

Fig. 3 HCA under $\mathrm{Vg}=\mathrm{Vd}$ is more than $\mathrm{HCA}$ under $\mathrm{Vg}=\mathrm{Vd} / 2$ for $\mathrm{L}=27 \mathrm{~nm}$.
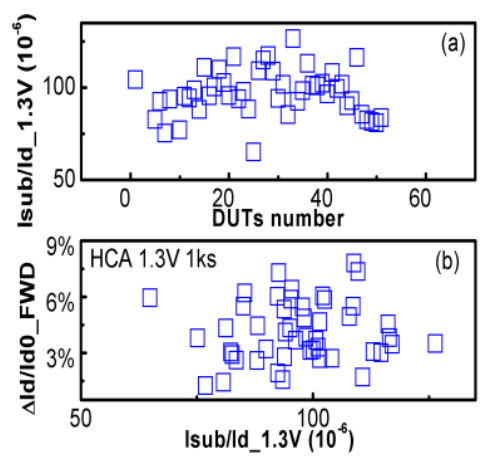

Fig. 7 Isub/Id does not represent HCA-stress well for nm-devices, as it has a device-to-device Fig.8 Although test data (o and $\square)$ show $(\Delta \mathrm{Id} / \mathrm{Id} \mathrm{F})<(\Delta \mathrm{Id} / \mathrm{Id}$ R), higher ' $\mathrm{n}$ ' for $\Delta \mathrm{Id} / \mathrm{Id} \mathrm{F} \quad$ Fig.9 The forward and reverse $\Delta$ Vth variation $(\mathrm{DDV})$ at stress $=0$ (a) and leads to incorrect $(\Delta \mathrm{Id} / \mathrm{Id} F)>(\Delta \mathrm{Id} / \mathrm{Id} R)$ when extrapolating. $\Delta \mathrm{Id} / \mathrm{Id} \mathrm{F}$ does not sense measured under $\mathrm{Vd}=0.1 \mathrm{~V}$ agrees its DDV does not correlate with the defects above space charges. The ' $\Delta$ ' in inset is calculated from (At ${ }^{0.29}$-Constant), well.

that of $\mathrm{HCA}$-induced $\Delta \mathrm{Id} / \mathrm{Id}$. which fits well with $\mathrm{Bt}^{0.34}$ (black line). Subtracting a constant from a real power law (red line) can give an 'apparent' higher ' $\mathrm{n}$ '. Id was measured under $\mathrm{Vg}=\mathrm{Vd}=0.9 \mathrm{~V}$.
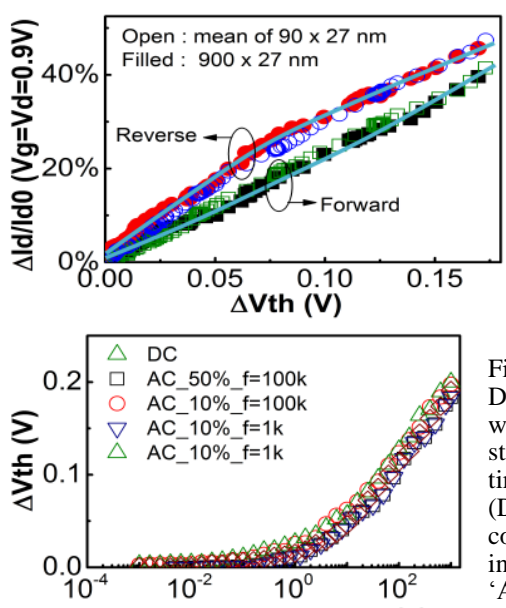

Equivalent stress time (s)

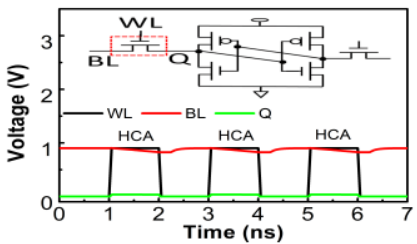

Fig. 4 The access nMOS in a SRAM during read-0 has a HCA duty factor of $\sim 50 \%$ (simulation).

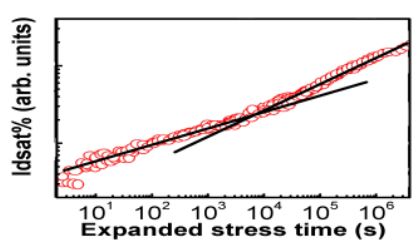

Fig.5 Variation of time exponent, ' $n$ ' (the line slope), with HCA time. A re-plot of data from ref. [2].

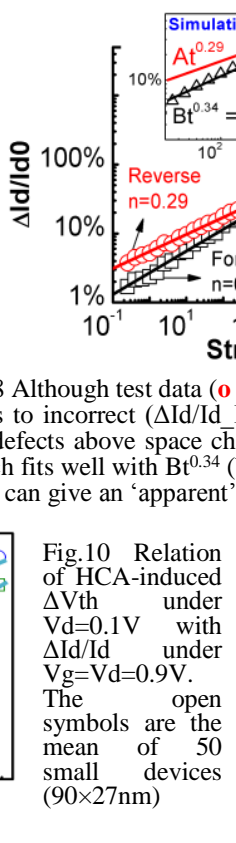

Fig.13 The AC and DC HCAs agree well when using equivalent stress time, i.e. time $\times$ Duty Factor (DF). The AC stress conditions are given in the format of and $\overline{\mathrm{V}} \mathrm{g}=\overline{\mathrm{V}} \mathrm{d}=2 \mathrm{~V}$.

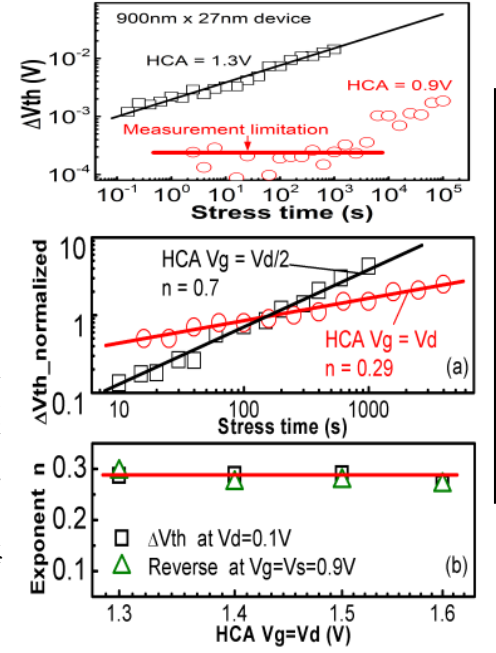

Fig. $11 \mathrm{HCA}$ is too low to establish kinetics reliably under use- $\mathrm{Vdd}=0.9 \mathrm{~V}$ and acceleration (e.g. $1.3 \mathrm{~V}$ ) is needed.

\section{Table 1:}

(1) $\Delta V t h=A \cdot t^{n} \cdot V_{g}{ }^{m}$

(2) $T_{\text {eff }}=T \cdot\left(\frac{V_{2}}{V_{1}}\right)^{m / n}$

(3) $A \cdot T^{n} \cdot\left(V_{2}\right)^{m}=A \cdot T_{\text {eff }}^{n} \cdot V_{1}{ }^{m} \quad\left(V_{2}>V_{1}\right)$

(4) $\mathrm{F}_{\mathrm{N}}\left(\Delta \mathrm{V}_{\mathrm{th}}, \eta\right)=\sum_{k=0}^{\infty} \frac{e^{-N} \mathrm{~N}^{k}}{k !} \mathrm{F}_{k}\left(\Delta \mathrm{V}_{\mathrm{th}}, \eta\right)$

(5) $\mathrm{N}=\frac{2 \mu^{2}}{\sigma^{2}} \quad \eta=\frac{\sigma^{2}}{2 \mu}$

(6) $\mu_{-} \Delta V t h=\mu_{-} L E_{-}+\mu_{-} W D F$

(7) $\sigma \_\Delta V t h=\left(\sigma_{-} L E^{2}+\sigma_{-} W D F^{2}\right)^{0.5}$

Fig. 12 (a) The time exponents under $\mathrm{Vg}=\mathrm{Vd}$ are smaller than that under $\mathrm{Vg}=\mathrm{Vd} / 2$. (b) The time exponent is insensitive to stress biases under $\mathrm{Vg}=\mathrm{Vd}$ 

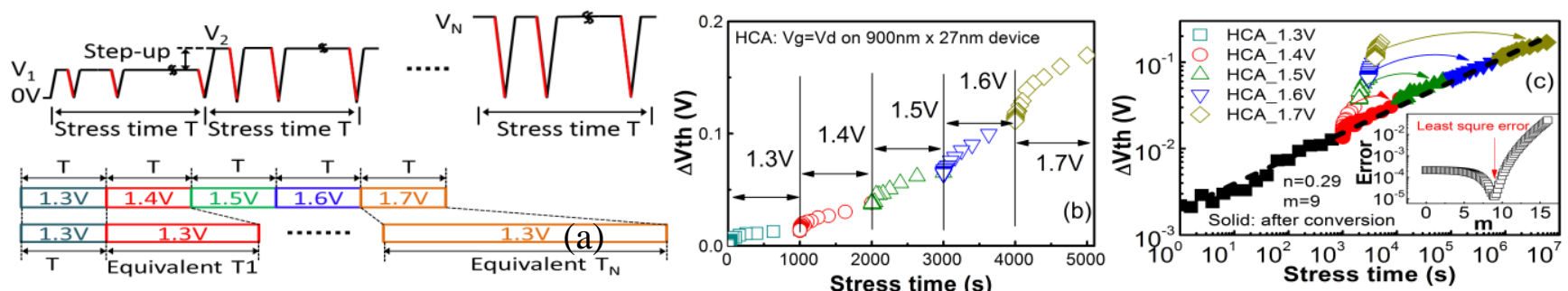

Fig.14 Voltage-Step-Stress (VSS) technique for HCA. (a) One device was stressed for a time $\mathrm{T}$ and the stress $\mathrm{Vg}=\mathrm{Vd}$ was then stepped up. $\Delta \mathrm{Vth}$ is plotted against linear (b) and $\log (\mathrm{c})$ stress time. The stress time under high bias is converted to an equivalent longer time at low bias by fitting the voltage exponent ' $\mathrm{m}$ ' (inset of (c)), based on Eqs.1-3 in Table 1. The dashed line has $\mathrm{n}=0.29$ and $\mathrm{m}=9$.

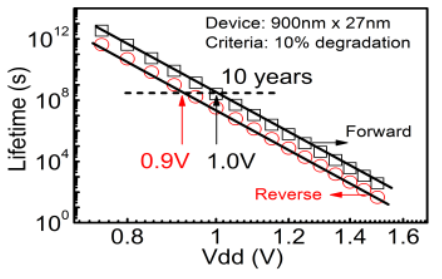

Fig.15 Evaluation of lifetime versus Vdd based on the model extracted from VSS tests in Fig.14.
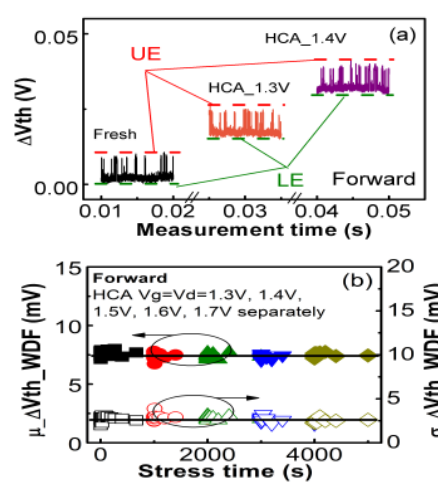

Fig. 18 (a) For $\mathrm{L} \times \mathrm{W}=27 \times 90 \mathrm{~nm}, \mathrm{LE}$ increases with $\mathrm{HCA}$, but $\mathrm{WDF}=\mathrm{UE}-$ LE does not. (b) The WDF mean of 50 devices and its sigma do not increase with stress time.
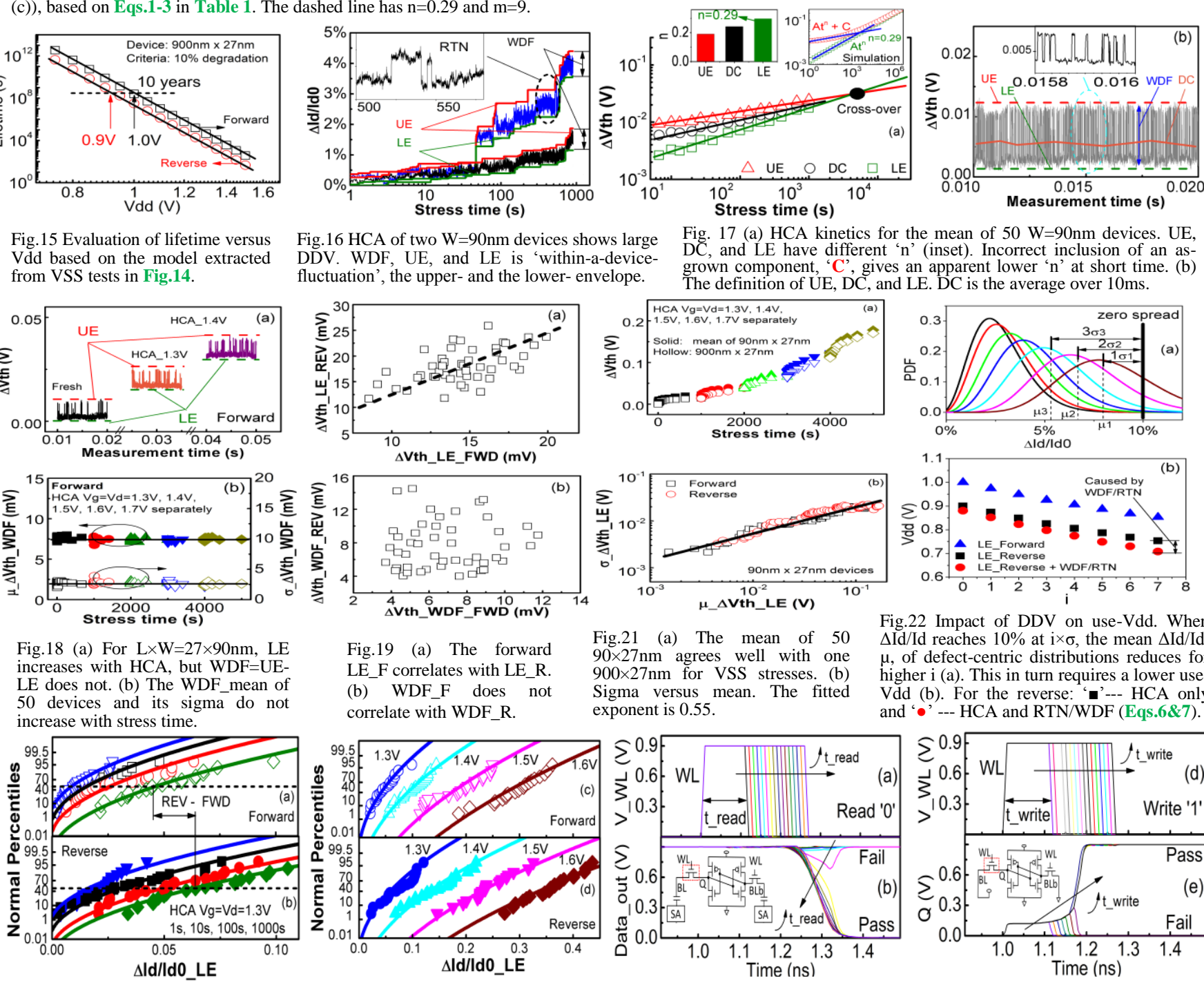

Fig. 16 HCA of two W=90nm devices shows large DDV. WDF, UE, and LE is 'within-a-devicefluctuation', the upper- and the lower- envelope.

Fig. 17 (a) HCA kinetics for the mean of $50 \mathrm{~W}=90 \mathrm{~nm}$ devices. UE, $D C$, and LE have different ' $n$ ' (inset). Incorrect inclusion of an asgrown component, ' $C$ ', gives an apparent lower 'n' at short time. (b) The definition of UE, DC, and LE. DC is the average over $10 \mathrm{~ms}$.
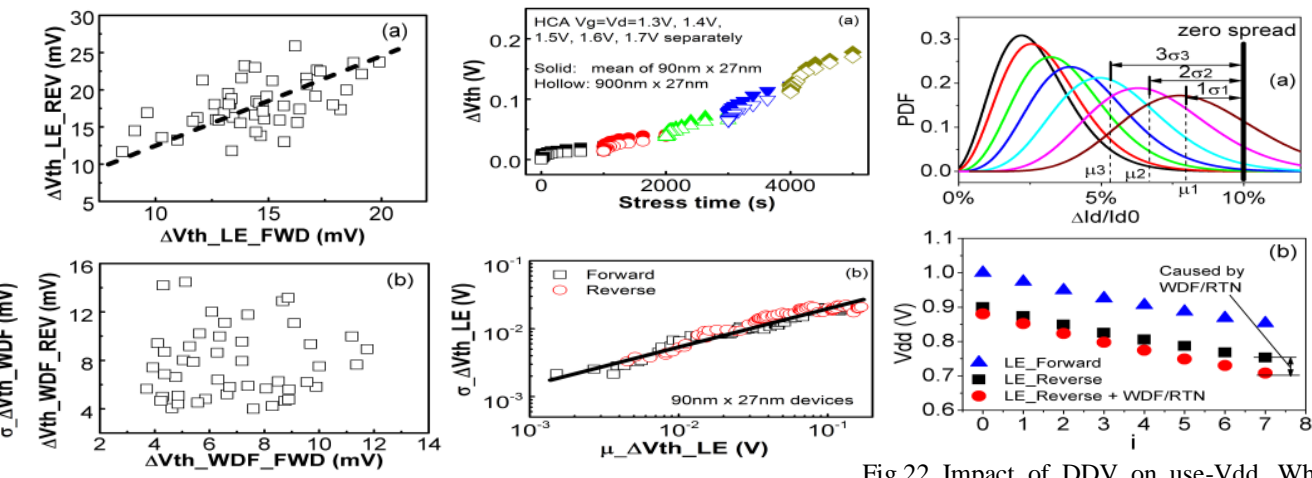

Fig.19 (a) The forward LE_F correlates with LE_R. (b) WDF_F does not correlate with WDF_R.

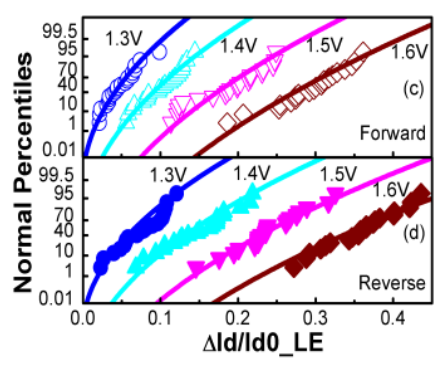

Fig.21 (a) The mean of 50 $90 \times 27 \mathrm{~nm}$ agrees well with one $900 \times 27 \mathrm{~nm}$ for VSS stresses. (b) Sigma versus mean. The fitted exponent is 0.55
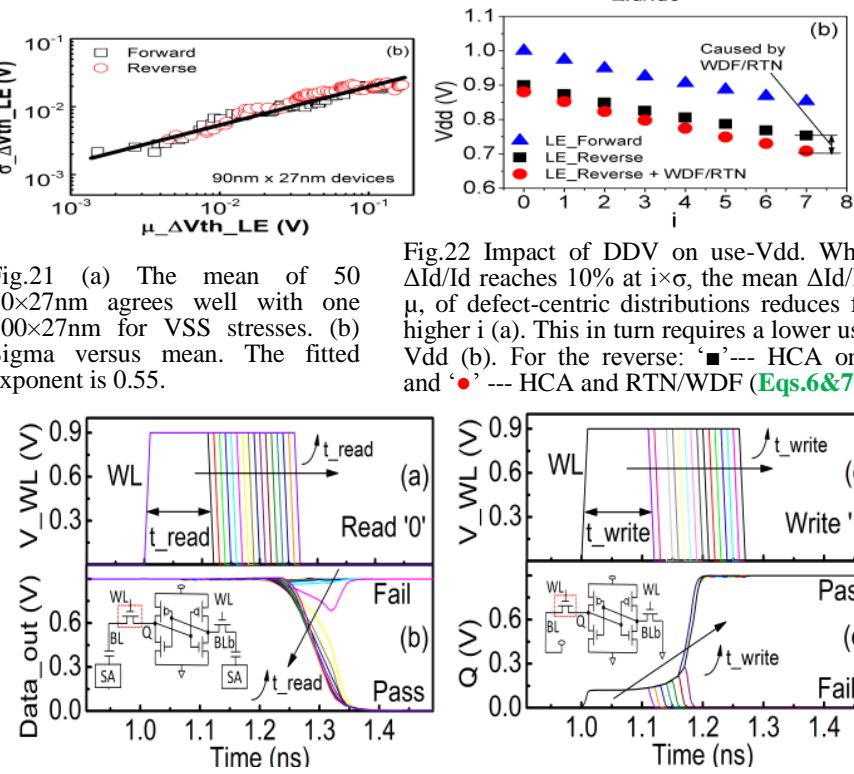

Fig.22 Impact of DDV on use-Vdd. When $\Delta \mathrm{Id} / \mathrm{Id}$ reaches $10 \%$ at $\mathrm{i} \times \sigma$, the mean $\Delta \mathrm{Id} / \mathrm{Id}$, $\mu$, of defect-centric distributions reduces for higher $\mathrm{i}(\mathrm{a})$. This in turn requires a lower useVdd (b). For the reverse: 'ø'--- HCA only and ' $\bullet---$ HCA and RTN/WDF (Eqs.6\&7).

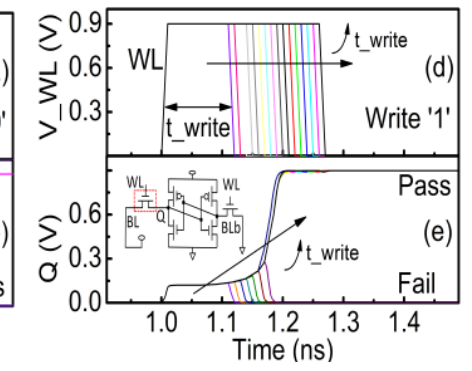

Fig.20 Statistics of LE DDV after different stress time (a\&b) and voltage (c\&d). The lines are fitted with the defect-centric distribution (Eqs.4\&5).
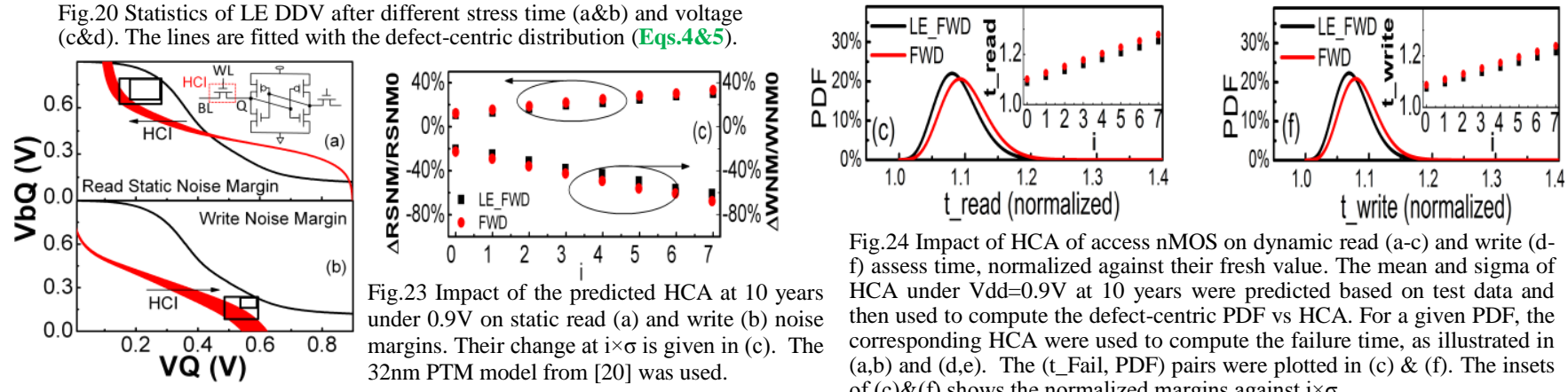

Fig.24 Impact of HCA of access nMOS on dynamic read (a-c) and write (df) assess time, normalized against their fresh value. The mean and sigma of HCA under $\mathrm{Vdd}=0.9 \mathrm{~V}$ at 10 years were predicted based on test data and then used to compute the defect-centric PDF vs HCA. For a given PDF, the corresponding HCA were used to compute the failure time, as illustrated in (a,b) and (d,e). The (t_Fail, PDF) pairs were plotted in (c) \& (f). The insets of (c)\&(f) shows the normalized margins against $i \times \sigma$. 